\title{
Phylogenetic Analysis of the Genus Corynebacterium Based on 16S rRNA Gene Sequences
}

\author{
CRISTINA PASCUAL, PAUL A. LAWSON, JOHN A. E. FARROW, MONTSERRAT NAVARRO GIMENEZ, \\ AND MATTHEW D. COLLINS* \\ Institute of Food Research, Reading Laboratory, Reading RG6 6BZ, United Kingdom
}

\begin{abstract}
The 16S rRNA gene sequences of 30 strains representing 23 validated Corynebacterium species and 7 currently non-valid Corynebacterium species were determined. These sequences were aligned with the sequences of other Corynebacterium species and related actinomycete taxa. A comparative sequence analysis revealed that there is considerable phylogenetic depth and internal structure in the genus Corynebacterium. Turicella otitidis and the amycolate species Corynebacterium amycolatum were located at the periphery of the genus Corynebacterium. It was evident that the species of the genus Corynebacterium form a monophyletic association and, together with other chemotype IV and mycolic acid-containing taxa (including the genera Dietzia, Gordona, Mycobacterium, Nocardia, Rhodococcus, and Tsukamurella), form a natural suprageneric group.
\end{abstract}

Originally, the genus Corynebacterium (Lehmann and Neumann 1896) was created essentially to accommodate the diphtheria bacillus and some other animal-pathogenic species (1). Later, however, an ill-assorted collection of other non-sporeforming, irregularly staining, gram-positive, rod-shaped species were assigned to this genus. The resulting genus not only encompassed phenotypically very diverse organisms but also included animal-pathogenic, plant-pathogenic, and saprophytic species $(4,6)$. The use of cellular chemical analysis, particularly from the 1960 s through the $1980 \mathrm{~s}$, did much to clarify the taxonomy of the corynebacteria $(7-9,17,24)$, and the genus is in the main now confined to those species which have wall chemotype IV (meso-diaminopimelic acid, arabinose, and galactose), contain corynemycolic acids (approximately 22 to 36 carbon atoms), cellular fatty acids of the straight-chain saturated and monounsaturated types, and dihydrogenated menaquinones, and have $\mathrm{G}+\mathrm{C}$ contents of approximately 51 to 68 mol\% $(6,19,26)$. This narrower definition of the genus Corynebacterium has resulted in the transfer of many species to other genera (e.g., the genera Arthrobacter, Curtobacterium, Clavibacter, Microbacterium, and Rhodococcus), as well as the assignment of species previously placed elsewhere to the genus Corynebacterium. However, there is still some evidence of heterogeneity within the genus Corynebacterium. For example, this genus includes both aerobic and facultatively anaerobic species (6); some species synthesize tuberculostearic acid (8); Corynebacterium bovis contains shorter and structurally distinctive mycolic acids (8); Corynebacterium amycolatum lacks mycolic acids (5); and so on. Thus, it is not known whether the members of the genus Corynebacterium as presently defined really do belong to a single genus.

Our knowledge of the intra- and intergeneric relationships of members of the genera Mycobacterium, Nocardia, and Rhodococcus and other long-chain mycolic acid-containing genera has improved greatly in recent years because of the advent of $16 \mathrm{~S}$ rRNA sequence analysis $(3,10,21-23,25)$. In particular, nucleotide sequence analysis has revealed that with the possible exception of the genus Nocardia, which branches off from within the radiation of the genus Rhodococcus (21, 22 ), the various long-chain mycolic acid-containing genera

* Corresponding author. Mailing address: Institute of Food Research, Reading Laboratory, Earley Gate, Reading RG6 6BZ, United Kingdom. Phone: 1734-357226. Fax: 1734-267917. form monophyletic groups. Furthermore, as a direct result of $16 \mathrm{~S}$ rRNA sequencing, the genus Gordona has been revived (25), and several new genera have been described, including the genera Dietzia (22) and Tsukamurella (10). To date, however, relatively few comparative $16 \mathrm{~S}$ rRNA sequence analyses have been performed with members of the genus Corynebacterium. Therefore, in this study we determined the almostcomplete $16 \mathrm{~S}$ rRNA gene sequences of 30 strains representing 23 validated Corynebacterium species and 7 nonvalid Corynebacterium species in order to (i) establish the intrageneric relationships within the genus Corynebacterium and (ii) determine whether the genus as currently defined is a monophyletic group.

\section{MATERIALS AND METHODS}

Cultures and cultivation. The strains which we examined are shown in Table 1. The strains were cultivated on nutrient agar supplemented with $5 \%$ sheep blood at 30 or $37^{\circ} \mathrm{C}$ for 18 to $24 \mathrm{~h}$.

Determination of 16S rRNA gene sequences. Genomic DNA was extracted from cells and purified by the method of Lawson et al. (18). A large fragment of the 16S rRNA gene was amplified by PCR by using universal primers pA (5'-AGAGTTTGATCCTGGCTCAG; positions 8 to 27 [Escherichia coli numbering]) and $\mathrm{pH}^{*}$ (5'-AAGGAGGTGATCCAGCCGCA; positions 1541 to 1522) as previously described (16). The PCR products were purified by using a Prep-A-Gene kit (Bio-Rad, Hercules, Calif.) according to the manufacturer's instructions and were sequenced by using a Taq Dye Deoxy terminator cycle sequencing kit (Applied Biosystems, Inc., Foster City, Calif.) and a model 373A automatic sequencer (Applied Biosystems, Inc.). In one case, Corynebacterium minutissimum, isolated PCR products were cloned directly into the $\mathrm{pCR}$ cloning vector (TA cloning kit; Invitrogen, San Diego, Calif.), which is supplied cut with T overhangs. Competent $E$. coli cells (One shot cells; Invitrogen) were transformed, and inserted fragments were sequenced by using standard Taq dyedeoxy terminator plasmid autosequencing methods.

Analysis of sequence data. The 16S rRNA gene sequences which we determined and previously published sequences of reference actinomycetes (obtained from the EMBL Data Library) were aligned by using the program PILEUP (12), and the alignments were corrected manually. Distance matrices were produced by using the program DNADIST of the PHYLIP package (13), and trees were constructed by the neighbour-joining method with the program NEIGHBOR of the same package. The stability of the groups was assessed by bootstrapping by using the programs SEQBOOT, DNADIST, NEIGHBOR, and CONSENSE (13). A total of 1,000 bootstrapped trees were generated. These analyses were performed by using a variety of different outgroups. In addition, a parsimony analysis (13) was performed with a reduced data set that included data for members of the genus Corynebacterium and data for Dietzia maris, Mycobactenium tuberculosis, and Tsukamurella paurometabola strains that were used reference organisms.

Nucleotide sequence accession numbers. The 16S rRNA gene sequences which we determined have been deposited in the EMBL Data Library under the accession numbers shown in Table 1. 
TABLE 1. Corynebacterium strains which we analyzed and their $16 \mathrm{~S}$ rRNA accession numbers

\begin{tabular}{lll}
\hline \multicolumn{1}{c}{ Species } & \multicolumn{1}{c}{ Strain" } & \multicolumn{1}{c}{ EMBL accession } \\
no.
\end{tabular}

${ }^{a}$ ATCC, American Type Culture Collection, Rockville, Md.; CIP, Collection of Institut Pasteur, Paris, France; DMMZ, Culture Collection of the Department of Medical Microbiology, University of Zürich, Zurich, Switzerland; NCDO, National Collection of Dairy Organisms, Reading, United Kingdom; NCFB, National Collection of Food Bacteria, Reading, United Kingdom; NCTC, National Collection of Type Cultures, London, United Kingdom; DSM, German Collection of Microorganisms, Braunschweig, Germany. $T=$ type strain.

\section{RESULTS}

The almost-complete $16 \mathrm{~S}$ rRNA sequences of 23 validated Corynebacterium species and 7 nonvalid Corynebacterium species were determined. All of the $16 \mathrm{~S}$ rRNA sequences except the $C$. minutissimum sequence were readily obtained by performing direct sequencing of PCR-amplified rRNA gene fragments. Direct sequencing of the $16 \mathrm{~S}$ rRNA genes of $C$. minutissimum proved to be problematical and resulted in extensive sequence terminations and ambiguities between approximately position 200 and position 450 . Therefore, $16 \mathrm{~S}$ ribosomal DNA products of $C$. minutissimum were cloned and sequenced, and this procedure revealed direct sequence analysis terminations derived from gene sequence microheterogeneity. Two distinct clones were detected when the sequence analysis was performed; one of these clones had an extra $(T)$ in region $V 2$, and the other contained an additional base $(\mathrm{T})$ in region $\mathrm{V} 3$, which resulted in extensive ambiguities when the direct PCR sequencing procedure was used.

The Corynebacterium strains which we examined and their EMBL 16S rRNA accession numbers are shown in Table 1. The sequences which we determined were aligned and compared with homologous sequences of more than 100 other high-G+C-content actinomycetes, including members of more than 70 representative species belonging to other mycolic acidcontaining genera (the genera Dietzia, Gordona, Mycobacte- rium, Nocardia, Rhodococcus, and Tsukamurella). In the comparative phylogenetic analysis approximately 100 bases were omitted from the $5^{\prime}$ end of the gene because of alignment ambiguities in hypervariable region V1. Levels of sequence similarity for an approximately 1,320-nucleotide region (ranging from position 107 to position 1438 [E. coli numbering]) were determined, and derived distance matrices (which were corrected by using Felsenstein's maximum-likelihood parameters) were used to elucidate the phylogenetic relationships of corynebacteria and their close relatives by the neighbor-joining method. A tree depicting the intra- and intergeneric phylogenetic relationships of members of the genus Corynebacterium, which was constructed by using a data set that included data for 57 other mycolic acid-containing strains and 27 non-mycolic acid-containing outgroup strains, is shown in Fig. 1. To ascertain whether a large number of outgroup strains resulted in distortions of the intrageneric relationships of Corynebacterium strains, the treeing analysis was repeated by using a reduced data set that included data for members of the genus Corynebacterium and three outgroup organisms (D. maris, $M$. tuberculosis, and T. paurometabola). All of the significant relationships inferred from the neighbor-joining data in the large data set (Fig. 1) were confirmed when the small data set was used. The intrageneric structure of the genus Corynebacterium was also investigated by parsimony analysis. Using this method and the reduced data set (data for members of the genus Corynebacterium and three outgroup strains), we obtained three equally parsimonious trees. All of the significant associations (Fig. 1) were confirmed and the trees exhibited differences for only three species (Corynebacterium variabilis, $C$. minutissimum, Corynebacterium striatum), whose branching patterns were known to be uncertain (as indicated by low bootstrap values).

\section{DISCUSSION}

Intrageneric relationships. Species currently considered members of the genus Corynebacterium sensu stricto (defined as organisms which have wall chemotype IV and contain corynemycolic acids), including C. amycolatum, exhibited levels of $16 \mathrm{~S}$ rRNA sequence similarity of $>91.5 \%$ with each other (Table 2). Both the distance calculations and the treeing analysis revealed that there is considerable phylogenetic structure within the genus Corynebacterium sensu stricto; some species formed distinct subgroups within the genus, while others formed individual lines.

Corynebacterium diphtheriae, the type species of the genus, formed a distinct subgroup with Corynebacterium kutscheri, Corynebacterium pseudotuberculosis, Corynebacterium vitarumen, and "Corynebacterium ulcerans." This subgroup was recovered in $99 \%$ of the bootstrapped trees. The type strains of $C$. diphtheriae and $C$. pseudotuberculosis exhibited a level of sequence similarity of $97.8 \%$, indicating that although these species are different, they are very closely related genetically. A previous report of phage-induced diphtheria toxin production (20) by strains of $C$. pseudotuberculosis is consistent with the observed close genealogical affinity of these species. The $16 \mathrm{~S}$ rRNA sequence of the single strain of the invalid species " $C$. ulcerans" examined was found to be almost identical (level of sequence similarity, $99.7 \%$, corresponding to five differences) to the sequence of the type strain of $C$. pseudotuberculosis. It is noteworthy that some strains of " $C$. ulcerans" are similar to $C$. pseudotuberculosis in that they produce phospholipase D and/or diphtheria toxin (2). On the basis of nonquantitative DNA hybridization data, Groman et al. (15) reported that although "C. ulcerans" and C. pseudotuberculosis are distinct, 


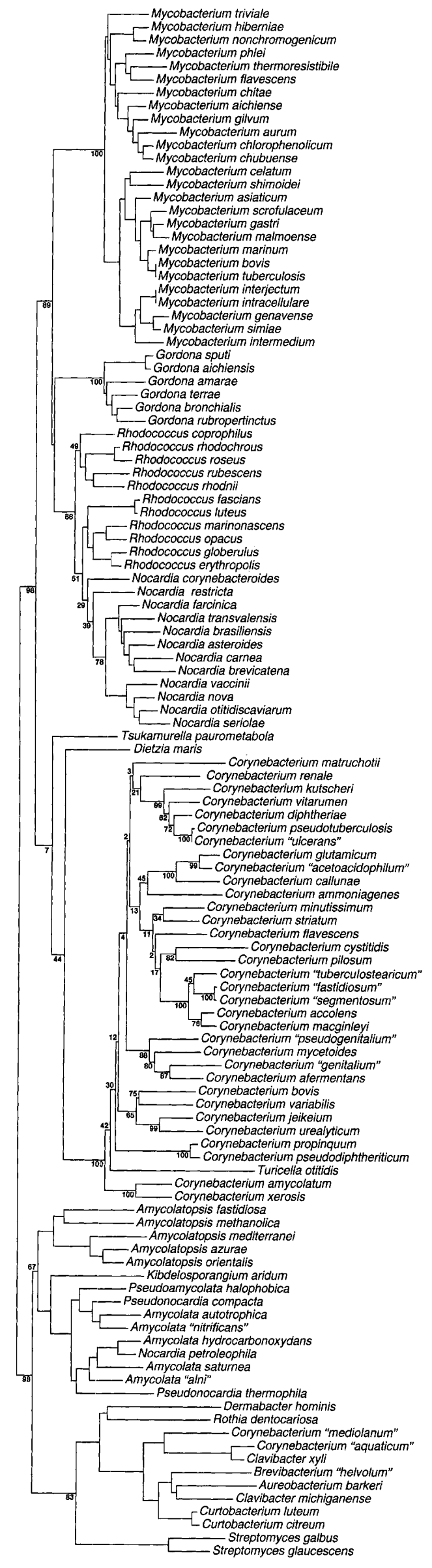

they are more closely related to each other than to $C$. diphtheriae. The observed levels of 16S rRNA sequence divergence of these three species are consistent with the findings of Groman et al. (15). Corynebacterium afermentans and "Corynebacterium genitalium" were found to be genealogically specifically related to each other (level of sequence similarity, approximately 97\%). These species, together with Corynebacterium mycetoides and Corynebacterium pseudogenitalium, formed a distinct subgroup (bootstrap value, $88 \%$ ) within the genus Corynebacterium. Corynebacterium jeikeium and Corynebacterium urealyticum exhibited a specific association (level of sequence similarity, approximately 97\%; bootstrap value 99\%), as did Corynebacterium pseudodiphtheriticum and Corynebacterium propinquum (level of sequence similarity, approximately $99 \%$; bootstrap value, $100 \%$ ). "Corynebacterium tuberculostearicum" formed a distinct subgroup with the invalid species "Corynebacterium fastidiosum" and "Corynebacterium segmentosum" (bootstrap value, 100\%) and the latter two organisms had almost identical sequences (level of sequence similarity, 99.9\%). The level of sequence divergence observed between the " $C$. fastidiosum"."C. segmentosum" group and " $C$. tuberculostearicum" (approximately $2 \%$ ) is strongly indicative of a new species, although DNA-DNA pairing studies will be necessary to determine whether " $C$. fastidiosum" and " $C$. segmentosum" are synonyms or separate species. Corynebacterium accolens and Corynebacterium macginleyi were specifically related to each other and to "C. tuberculostearicum," "C. fastidiosum," and " $C$. segmentosum" (bootstrap value for the group, 100\%). The three glutamic acid-producing species, "Corynebacterium acetoacidophilum," Corynebacterium callunae, and Corynebacterium glutamicum, formed a distinct subgroup, which is consistent with the close phenotypic resemblance of these organisms (19). Corynebacterium cystitidis and Corynebacterium pilosum, which once were considered biotypes of Corynebacterium renale, were also recovered together, and the bootstrap analysis (bootstrap value, $82 \%$ ) revealed that the association of these taxa is probably significant. The type strains of $C$. amycolatum and Corynebacterium xerosis were found to be genealogically closely related (level of sequence similarity, approximately 97\%). Although this association between $C$. amycolatum and $C$. xerosis is significant (bootstrap value, 100\%), it was unexpected, as the former species differs from other Corynebacterium species in lacking mycolic acids (5). Unlike C. amycolatum, $C$. xerosis has not been considered an exceptional or unusual member of the genus Corynebacterium. It should be noted, however, that $C$. xerosis is phenotypically and genotypically heterogeneous (11), and how representative the type strain of this species is, remains open to question.

All other species formed individual sublines which were in the main defined by low bootstrap values (often less than $40 \%$ ). The branching position or order of these species is relatively uncertain and can be expected to change to some extent with the nature of the reference species and/or analytical methods used. One of these individual sublines, the species C. bovis, is particularly noteworthy (6). The mycolic acids of $C$. bovis differ markedly from the mycolic acids of other Corynebacterium species (e.g., they have lower molecular weights) (7), and consequently classification of $C$. bovis in the genus Corynebacterium is considered uncertain $(6,26)$. It is apparent from the results of the treeing analysis, however, that $C$. bovis clusters within the confines of the genus Corynebacterium sensu stricto and should be considered an authentic member of the genus.

FIG. 1. Phylogenetic dendrogram based on a comparison of the 16S rRNA sequences of Corynebacterium species and some close relatives. The branching pattern was generated by the neighbor-joining method, and bootstrap values (indicated at the nodes) were calculated from 1,000 trees. 


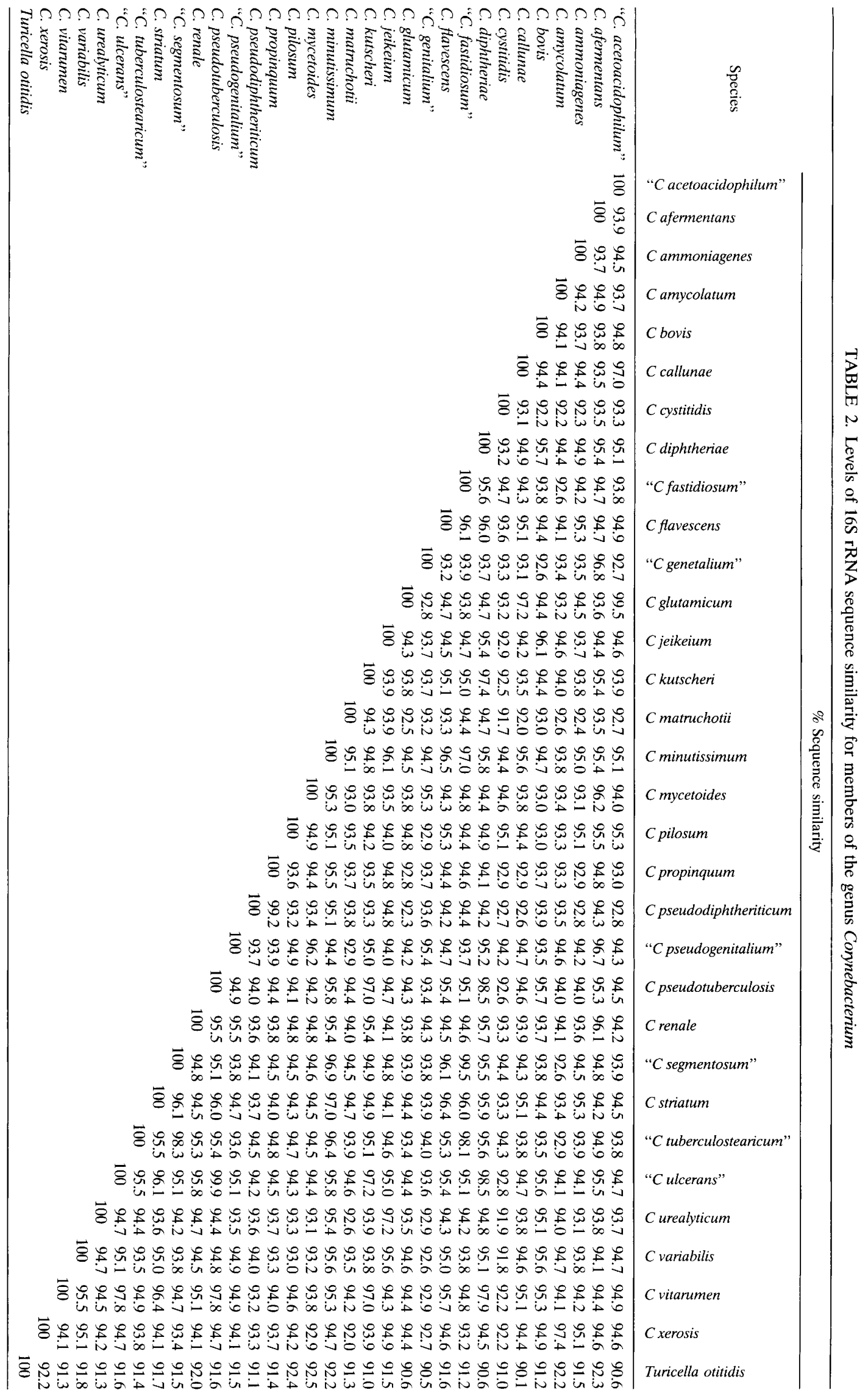


Position of the amycolate species $C$. amycolatum and Turicella otitidis. C. amycolatum and C. xerosis appear to form the deepest subline within the genus Corynebacterium. The recently described species Turicella otitidis (14) also forms a relatively long subline branching from a position close to the periphery of the genus. Both $C$. amycolatum and Turicella otitidis are known to lack mycolic acids $(5,14)$. Turicella otitidis is also purported (14) to differ from authentic corynebacteria in possessing unsaturated menaquinones (true corynebacteria invariably synthesize dehydrogenated menaquinones) (6). Because of these chemotaxonomic differences it could be argued that both of these taxa should not be considered members of the genus Corynebacterium sensu stricto. Although the exact branching positions of $C$. amycolatum and Turicella otitidis (as indicated by low bootstrap values) are somewhat uncertain, it is evident from tree topology considerations that the exclusion of these taxa from the genus Corynebacterium would also mean that $C$. xerosis must be removed. The position of $C$. xerosis clearly merits investigation in view of the deep phylogenetic position of this taxon and its association with $C$. amycolatum.

Intergeneric relationships. Although the genus Corynebacterium sensu stricto (including C. amycolatum and C. xerosis) exhibits considerable phylogenetic depth (level of sequence divergence, approximately $8.5 \%$ ), with the exception of the above-mentioned species which could possibly be excluded from the genus, no simple subdivision of the genus Corynebacterium seems possible without an undesirable and phenotypically unjustifiable proliferation of new genera. It is clear from the treeing analysis results that species of the genus Corynebacterium form a monophyletic group that is separate from but equivalent in rank to other mycolic acid-containing genera (e.g., the genera Mycobacterium and Gordona). With the exception of Turicella otitidis, which clusters within the boundaries of the genus Corynebacterium, the closest phylogenetic relative of the corynebacteria is $D$. maris (formerly Rhodococcus maris). The position of $D$. maris between members of the genus Corynebacterium and members of the Rhodococcus-Nocardia-Mycobacterium-Gordona cluster (Fig. 1) is consistent with the data of Rainey et al. $(21,22)$. As found in previous studies (10,21-23), with the exception of the genus Nocardia, which branches off from within the radiation of the genus Rhodococcus, other mycolic acid-containing genera form monophyletic groups (Fig. 1).

It is now well documented (6) that members of the genus Corynebacterium have many of the same chemical markers as other mycolic acid-containing taxa; for example, they contain a directly cross-linked murein based on meso-diaminopimelic acid, a wall arabinogalactan polymer, mycolic acids, and nonhydroxylated fatty acids of the straight-chain saturated and monounsaturated types, some species also synthesize 10-methyl-branched acids, and most taxa contain phosphatidylinositol and phosphatidylinositol mannosides. The results of our phylogenetic analysis correlate well with these chemotaxonomic similarities and indicate that the genus Corynebacterium, together with other chemotype IV and mycolic acid-containing taxa (including the genera Dietzia, Gordona, Mycobacterium, Nocardia, Rhodococcus, and Tsukamurella), form a natural suprageneric association which, if it were at the rank of family, could be designated the family Mycobacteriaceae.

\section{ACKNOWLEDGMENTS}

This work was supported in part by grants ERBCHRX-CT 93-0194 and BI02-CT94-3098 from the European Community.

\section{REFERENCES}

1. Barksdale, L. 1970. Corynebacterium diphtheriae and its relatives. Bacteriol Rev. 34:378-422.

2. Carne, H. R., and E. O. Onan. 1982. The exotoxins of Corynebacterium ulcerans. J. Hyg. 88:173-191.

3. Chun, J., and M. Goodfellow. 1995. A phylogenetic analysis of the genus Nocardia with 16S rRNA gene sequences. Int. J. Syst. Bacteriol. 45:240-245.

4. Collins, M. D., and J. F. Bradbury. 1986. Plant pathogenic species of Corynebacterium, p. 1276-1283. In P. H. A. Sneath, N. S. Mair, M. E. Sharpe, and J. G. Holt (ed.), Bergey's manual of systematic bacteriology, vol. 2. The Williams \& Wilkins Co., Baltimore.

5. Collins, M. D., R. A. Burton, and D. Jones. 1988. Corynebacterium amycolatum sp. nov., a new mycolic acid-less Corynebacterium species from human skin. FEMS Microbiol. Lett. 49:349-352.

6. Collins, M. D., and C. S. Cummins. 1986. Genus Conyebacterium Lehmann and Neumann, $1896,350^{\mathrm{AL}}$, p. $1266-1276$. In P. H. A. Sneath, N. S. Mair, M. E. Sharpe, and J. G. Holt (ed.), Bergey's manual of systematic bacteriology, vol. 2. The Williams \& Wilkins Co., Baltimore.

7. Collins, M. D., M. Goodfellow, and D. E. Minnikin. 1982. A survey of the structures of mycolic acids in Corynebacterium and related taxa. J. Gen. Microbiol. 128:129-149.

8. Collins, M. D., M. Goodfellow, and D. E. Minnikin. 1982. Fatty acid composition of some mycolic acid-containing coryneform bacteria. J. Gen. Microbiol. 128:2503-2509.

9. Collins, M. D., T. Pirouz, M. Goodfellow, and D. E. Minnikin. 1977. Distribution of menaquinones in actinomycetes and corynebacteria. J. Gen. Microbiol. 100:221-230.

10. Collins, M. D., J. Smida, M. Dorsch, and E. Stackebrandt. 1988. Tsukamurella gen. nov. harboring Corynebacterium paurometabolum and Rhodococcus aurantiacus. Int. J. Syst. Bacteriol. 38:385-391.

11. Coyle, M. B., R. B. Leonard, D. J. Nowowiejski, A. Malekniazi, and D. J. Finn. 1993. Evidence of multiple taxa within commercially available reference strains of Corynebacterium xerosis. J. Clin. Microbiol. 31:1788-1793.

12. Devereux, J., P. Haeberli, and D. Smithies. 1984. A comprehensive set of sequence analysis programs for the VAX. Nucleic Acids Res. 12:387-395.

13. Felsenstein, J. 1989. PHYLIP - phylogeny inference package (version 3.2) Cladistics 5:164-166.

14. Funke, G., S. Stubbs, M. Altwegg, A. Carlotti, and M. D. Collins, 1994. Turicella otitidis gen. nov., sp. nov., a coryneform bacterium isolated from patients with otitis media. Int. J. Syst. Bacteriol. 44:270-273.

15. Groman, N., J. Schiller, and J. Russell. 1984. Corynebacterium ulcerans and Corynebacterium pseudotuberculosis responses to DNA probes derived from corynephage $\beta$ and Connebacterium diphtheriae. Infect. Immun. 45:511-517.

16. Hutson, R. A., D. E. Thompson, and M. D. Collins. 1993. Genetic interrelationships of saccharolytic Clostridium botulinum types B, E and F and related clostridia as revealed by small-subunit rRNA gene sequences. FEMS Microbiol. Lett. 108:103-110.

17. Keddie, R. M., and G. L. Cure. 1977. The cell wall composition and distribution of free mycolic acids in named strains of coryneform bacteria and in isolates from various natural sources. J. Appl. Bacteriol. 42:229-252.

18. Lawson, P. A., S. E. Gharbia, H. N. Shah, and D. R. Clark. 1989. Recognition of Fusobacterium nucleatum subgroups Fn-1, Fn-2 and Fn-3 by ribosomal RNA gene restriction patterns. FEMS Microbiol. Lett. 65:41-46.

19. Liebl, W. 1991. The genus Corynebacterium-nonmedical, p. 1157-1171. In A. Balows, H. G. Truper, M. Dworkin, W. Harder, and K. H. Schleifer (ed.), The prokaryotes, 2nd ed. Springer-Verlag, New York.

20. Maximescu, P., A. Oprisan, A. Pop, and E. Potorac. 1974. Further studies on corynebacterium species capable of producing diphtheria toxin $(C$. diphtheriae, C. ulcerans, C. ovis). J. Gen. Microbiol. 82:49-56.

21. Rainey, F. A., J. Burghardt, R. M. Kroppenstedt, S. Klatte, and E. Stackebrandt. 1995. Phylogenetic analyses of the genera Rhodococcus and Nocardia and evidence for the evolutionary origin of the genus Nocardia from within the radiation of Rhodococcus species. Microbiology 141:523-528.

22. Rainey, F. A., S. Klatte, R. M. Kroppenstedt, and E. Stackebrandt. 1995. Dietzia, a new genus including Dietzia maris comb. nov., formerly Rhodococcus maris. Int. J. Syst. Bacteriol. 45:32-36.

23. Ruimy, R., P. Boiron, V. Boivin, and R. Christen. 1994. A phylogeny of the genus Nocardia deduced from the analysis of small-subunit ribosomal DNA sequences, including transfer of Nocardia amarae to the genus Gordona as Gordona amarae comb. nov. FEMS Microbiol. Lett. 123:261-268.

24. Schleifer, K. H., and O. Kandler. 1972. Peptidoglycan types of bacterial cell walls and their taxonomic implications. Bacteriol. Rev. 36:407-477.

25. Stackebrandt, E., J. Smida, and M. D. Collins. 1989. Evidence of phylogenetic heterogeneity within the genus Rhodococcus: revival of the genus Gordona (Tsukamura). J. Gen. Appl. Microbiol. 34:341-348.

26. Von Graevenitz, A., and T. Krech. 1991. The genus Corynebacterium-medical, p. 1172-1187. In A. Balows, H. G. Truper, M. Dworkin, W. Harder, and K. H. Schleifer (ed.), The prokaryotes, 2nd ed. Springer-Verlag, New York. 